\title{
Evolution is Linear: Debunking Life's Little Joke
}

\author{
Ronald A. Jenner \\ Department of Life Sciences, The Natural History Museum, Cromwell Road, SW7 \\ 5BD London, UK \\ *Corresponding author \\ Ronald A. Jenner \\ E-mail: r.jenner@nhm.ac.uk
}

\section{An infamous icon}

The linear march of progress from apish ancestor to modern human is instantly recognisable, endlessly parodied, but also universally rejected by scientists as an accurate depiction of evolution (Fig. 1). The most iconic version of the linear depiction of evolution appeared in Francis Clark Howell's 1965 book Early Man [1]. Biologist David Archibald expresses scientific consensus when he writes that "This kind of representation fuels profound misunderstandings by the general public of how evolution operates" leading to "misperceptions of evolution as a straight-line, progressive process" [2: 19]. Archibald argues that linear evolutionary imagery reflects the conceptual stain of the scala naturae, which continues to besmirch evolutionary thinking in popular culture.

Archibald [2: 19] called the continuing prevalence of the ladder of life "Steve Gould's Bane". Stephen Jay Gould was the most widely read palaeontologist of the last half century, and he indefatigably battled the linear depiction of evolution in his popular and professional writing. Gould dubbed the mistaken impression that evolution is linear "Life's Little Joke" because its clearest examples are a few unsuccessful taxa that were decimated by extinction to a single surviving genus or species. The resulting iconography is a ladder from past to present, with horse and human evolution as classic examples. In reality, Gould argues, evolution branches and produces a bushlike genealogy, and "we can linearize a bush only if it maintains but one surviving twig that we can falsely place at the summit of a ladder" [3: 181].

Gould's career-long campaign to substitute bushes for ladders as evolution's proper metaphor has been immensely influential. Unfortunately, it is also conceptually flawed, and has caused widespread confusion in popular, educational, and even professional literature. A triple anniversary makes 2017 a timely year to debunk Life's Little Joke: the $10^{\text {th }}$ anniversary of the death of Francis Clark Howell, whose book brought us the iconic image of linear human evolution; the $15^{\text {th }}$ anniversary of the death of Stephen Jay Gould, the icon's most vociferous and insistent critic; and the $30^{\text {th }}$ anniversary of Gould's essay that gave this perceived conceptual evil its name.

\section{Linear evolution rejected}

Gould rejected linear evolutionary imagery in several publications. I focus here exclusively on the 1987 essay titled "Life's Little Joke" (I cite from the reprinted version in Bully for Brontosaurus) where he illustrates it with horse evolution. The 
fossil record shows that the tree of horses was once much more diverse than the single surviving genus Equus. Gould accepts that Hyracotherium was the ancestor of all horses, and writes:

"To be sure, Hyracotherium is the base of the trunk (as now known), and Equus is the surviving twig. We can, therefore, draw a pathway of connection from a common beginning to a lone result. But the lineage of modern horses is a twisted and tortuous excursion from one branch to another, a path more devious than the road marked by Ariadne's thread from the Minotaur at the center to the edge of our culture's most famous labyrinth. Most important, the path proceeds not by continuous transformation but by lateral stepping" [3: 175].

Gould paints an incredibly misleading picture of evolution here. Evolutionary lineages are not twisted, tortuous and labyrinthine. If the pathway from

Hyracotherium to Equus is an ancestor-descendant lineage it is straight. Tracing this lineage forwards or backwards in time involves navigating a number of turns along internal branches, but such bends are just pictorial constraints of drawing a tree. All internal branches between each terminal taxon and the root of the tree form an unbroken, unbranched lineage of ancestors and continuous evolutionary transformation through time. In the absence of processes that cause the horizontal transfer of genetic information between lineages, such as hybridisation, horizontal gene transfer, or symbiogenesis, evolution is linear (I ask readers to keep this caveat in mind). Without these processes, which Gould doesn't discuss, evolution by lateral stepping is nonsense, as is the bizarre suggestion that a lineage "hops from branch to branch through a phylogenetic bush" [3: 180].

Gould concludes that palaeontologist George Gaylord Simpson was incarcerated in the straitjacket of the scala naturae for believing in the importance "of evolution by transformational change within populations rather than by accumulation across numerous events of discrete, branching speciation" [3: 178]. But this suggests a false opposition of the linearity of descent and the bushiness that results from speciation. The linear and branching aspects of evolution are inextricably linked, with each terminal branch tipping a lineage of common ancestors shared with other taxa. By falsely dichotomizing the linear and branching aspects of evolution as rival perspectives Gould sowed the seeds for enormous misunderstanding in unsuspecting readers.

Remarkably, the same views are on display in Gould's professional writing. For instance, in his 1988 Presidential Address to the Paleontological Society he again portrays the linear and branching aspects of evolution as conflicting viewpoints, and disapprovingly concludes that although we now "recognize the bushiness of equid and hominid trees... [we] still view the lone survivors as end-products of a coherent anagenetic sequence within the bush" [italics in original] [4: 319]. But how could it be otherwise? Every terminal twig in a tree is unique because of evolution within each lineage after it diverged from its relatives.

Gould's wasn't the only prominent voice who tried to eradicate linear evolutionary thinking. In the year of Gould's Presidential Address, Robert O'Hara published an influential paper in which he coined the now ubiquitous phrase "tree thinking" to explain the importance of cladistic principles for evolutionary biology [5]. According to O'Hara proper three thinking banishes linear evolutionary narratives because the central subjects that provide these narratives with unity and continuity through time 
are clades, and because clades are branching, our evolutionary narratives should branch as well. But clades aren't the central subjects of evolutionary narratives, lineages are. Clades are just bundles of diverging lineages. O'Hara wrongly claims that we are imposing "Artifical closure" [5: 152] upon evolutionary narratives by making them linear. The story of the evolution of any given trait, taxon or clade can be captured in a linear narrative because each perches on top of a linear string of ancestors.

\section{Misdiagnosing the sin of the scala}

Tree thinking has eradicated much incorrect linear evolutionary thinking. Its proper target is the phylogenetic version of the scala naturae: the misinterpretation of a linear arrangement of collateral relatives (extant or extinct) as an evolutionary series of ancestors and descendants. The systematic relationships between collateral relatives is the realm of evolutionary branching, but this branched pattern is produced by the linear process of descent with modification within ancestor-descendant lineages. Speciation just produces more independently evolving lineages. Put another way, the branching realm of relationships between collateral relatives has no time dimension (it is synchronic), while the realm of ancestor-descendant lineages extends linearly through time (it is diachronic). Linearly connecting taxa in the synchronic realm of systematic relationships is to commit the sin of the scala, and this is why you should not read a tree across its tips. But mistaking the sin of the scala with the diachronic linearity of evolving lineages continues to drive the witch-hunt against linear evolutionary imagery.

Worryingly, this misguided crusade is prominent in the educational literature. A paper by Catley et al. [6] in the Journal of Research in Science Teaching that cites both Gould and O'Hara is symptomatic. They conclude that evolution should not be taught with diagrams that place fossils along internal branches because this implies "one taxon turning into a different taxon over time" [6: 18], which is "contrary to the accepted explanation of evolution" [6: 8] as happening via branching. But linear descent and lineage branching are not in conflict. Internal branches represent lineages of real evolving taxa. The reason why we place fossils so rarely on internal branches is due entirely to the epistemological challenge of recognizing fossils as ancestors even when they are. Catley et al. are unnecessarily worried about the prevalence of linear transformational thinking among students. The problem is that their exclusive cladistic focus on branching patterns of collateral relatives ignores the deeper linearity of evolving lineages, and misrepresents how systematic pattern and evolutionary process are connected.

This misunderstanding is concisely expressed in an online resource for science teachers hosted by Indiana University Bloomington: "As teachers, we often encounter the misconception that ancestral relationships are lineal, when in fact family trees and phylogenies are branched" [bold in original]" [7]. Other tree thinkers seem similarly blind to the diachronic aspect of evolution.

Biologist Ryan Gregory published a paper that is explicitly indebted to the ideas of Gould and O'Hara titled "Understanding evolutionary trees" in the journal Evolution: Education and Outreach [8]. It contains a cladogram of extant primate relationships, with monkeys being paraphyletic with respect to a clade of the great apes and 
humans. Nevertheless, Gregory concludes that we didn't descend from monkeys, "and no sane biologist suggests otherwise" [8: 132]. Yet, his tree suggests we did evolve from monkeys, but these monkeys were extinct. By omitting this small linguistic detail, which carries enormous conceptual baggage, Gregory creates a misleading paradox between his text and figure. The denial of our monkey ancestry is a widespread canard in the popular and educational literature and rooted in a similar lack of conceptual precision. In his book, dedicated to Gould, on the importance of fossils for understanding evolution Donald Prothero tries to slay linear evolutionary imagery by invoking the false dichotomy that "Evolution is a bush, not a ladder!" [italics and exclamation mark in original] [9: 125]. This sets the stage for Prothero concluding that thinking "we come from monkeys is simply wrong" [9: 347], and that believing so would be "to live in a web of lies" [9: 347], despite presenting a primate tree identical to the one in Gregory, and concluding earlier in the book that we still "have the genes for the long tails of our monkey ancestors" [9: 98].

Prothero fails to grasp the proper relationship of the synchronic and diachronic realms of evolution when he invokes the nonsensical concept of "collateral ancestors" [9: 82]. He defines these as organisms that share common ancestry with another taxon, but which are not directly ancestral to it. But this makes collateral ancestors collateral relatives, and relatives aren't ancestors. This concept confuses the realms of linear evolutionary descent and branching systematic pattern. Finally, consider Ian Tattersall, who considers Gould's pushing of the bush to have been "a most seminal contribution to paleoanthropology" [10: 118]. Tattersall approvingly cites from a 1976 essay where Gould espouses the view that "'ladders' (evolution as a continuous sequence of ancestors and descendants) do not represent the path of evolution" [10: 119]. I don't know what evolutionary descent is if not a continuous sequence of ancestors and descendants.

\section{Gould's blind spot and the cladistic blindfold}

Throughout his career Gould tried to demarcate sovereign territory for a macroevolutionary theory that didn't just flow smoothly from microevolutionary processes. But by trying to shift the engine of evolutionary change from evolving populations to the higher-level of the waxing and waning of species, I think he lost sight of the fundamental linearity of descent that ties together micro- and macroevolution.

A shallow cladistic focus on the branching relationships of sister taxa can likewise mask the deep linearity of evolving lineages. The connection between linear process and branched product is not intuitive. The linear schemes drawn by early evolutionists and untrained students today bear witness to this conceptual challenge. The almost universal rejection of linear evolutionary imagery to depict the evolutionary process is misplaced. Evolutionary descent is linear. But largely as a result of Gould's flawed arguments and naïve tree thinking many seem to have forgotten this basic truth.

Pernicious evolutionary notions, such as predictable unidirectional progress, goaldirectedness, anthropocentrism, or evolution following a main line can all be exterminated by rejecting the linear view of evolution, as Gould did routinely. But linearity per se is the wrong target. Even the sin of the scala isn't wrong because it is linear, but because it lines up the wrong entities. 
Figure 1. A version of the iconic linear march of human evolution. Linear evolutionary imagery like this is ubiquitous and universally condemned as offering a distorted picture of the evolutionary process. However, as a bare-bones metaphor of the process of descent with modification the image is accurate when interpreting the five leftmost figures as inferred or imagined ancestors. Only when the depicted figures are collateral relatives rather than ancestors does such imagery promote an incorrect view of evolution. It is noteworthy that most published images of linear evolution do not specify what the figures are, which tempts readers to interpret them as ancestors. (From

https://commons.wikimedia.org/wiki/File:Human_evolution_scheme.svg).

\section{References}

$1 \quad$ Howell FC. 1965 . Early Man. New York: Time-Life.

2 Archibald JD. 2014. Aristotle's ladder, Darwin's tree. The evolution of visual metaphors for biological order. New York: Columbia University Press.

3 Gould SJ. 1991. Bully for brontosaurus. London: Penguin Books.

4 Gould SJ. 1988. Trends as changes in variance: a new slant on progress and directionality in evolution. 62: 319-29.

5 O'Hara RJ. 1988. Homage to Clio, or, toward an historical philosophy for evolutionary biology. Syst Zool 37: 142-55.

6 Catley KM, Novick LR, Shade CK. 2010. Interpreting evolutionary diagrams: when topology and process conflict. J Res Sci Teach 47: 861-82.

7 Flammer L, Beard J, Nelson CE, Nickels M. 2017. ENSIWEB.

Evolution/Nature of Science Institutes. Retrieved on 13 October 2017 from http://www.indiana.edu/ ensiweb/phylo. tree.html.

8 Gregory TR. 2008. Understanding evolutionary trees. Evo Edu Outreach 1: 121-37.

9 Prothero DR. 2007. Evolution. What the fossils say and why it matters. New York: Columbia University Press.

10 Tattersall I. 2013. Stephen J. Gould's intellectual legacy to anthropology. In Danieli GA, Minelli A, Pievani T, eds; Stephen J Gould: The scientific legacy. Milan: Springer Verlag. p 115-27. 